\title{
Optimised Multi-Electrode Topology for Piezoelectric Material Characterisation
}

\author{
Leander Claes ${ }^{1}$, Nadine Feldmann ${ }^{1}$, Benjamin Jurgelucks², Veronika Schulze ${ }^{2}$, \\ Stephan Schmidt ${ }^{2}$, Andrea Walther ${ }^{2}$, Bernd Henning ${ }^{1}$ \\ ${ }^{1}$ Measurement Engineering Group, Paderborn University, Warburger Straße 100, \\ 33098 Paderborn, Germany \\ ${ }^{2}$ Mathematical Optimization, Humboldt-Universität zu Berlin, Rudower Chaussee 25, \\ 12489 Berlin, Germany \\ claes@emt.uni-paderborn.de
}

\section{Summary:}

The increase in availability of computational resources resulted in the advent of simulation-driven design of sensor and actor systems. A prerequisite of this design process is a sufficiently accurate and complete set of material parameters. When it comes to piezoelectric materials, these data sets are especially hard to acquire, as they must include the mechanical, electrical and piezoelectric properties, resulting in a minimum of ten material parameters to be determined. In this work, the authors describe an approach of designing a test specimen with an optimised electrode topology that is to be used in a material characterisation procedure using only electrical impedance measurements. The aim is to increase the sensitivity of the measured impedance with respect to each of the material parameters, so that these parameters can be identified from an electrical impedance measurement in an inverse procedure.

Keywords: Piezoelectricity, material parameters, optimisation, topology, inverse procedure

\section{Motivation}

Identification of piezoelectric material parameters is typically done by applying the well-known IEEE Standard on Piezoelectricity [1]. However, this standard requires measurement on four differently conditioned specimens for the identification of a full set of material parameters. As different processing procedures are required for each of those specimens, they are prone to have different material parameters, which in turn will lead to inconsistencies in the determined data set. An alternative approach using a single disc-shaped specimen with a three-electrode setup is developed by Feldmann et al., which shows that a full set of piezoelectric parameters (the elements of the stiffness matrix $c_{11}, c_{12}, c_{13}, c_{33}$ and $c_{44}$, of the permittivity matrix $\varepsilon_{11}$ and $\varepsilon_{33}$ and of the piezoelectric coupling matrix $e_{15}, e_{31}$ and $e_{33}$ ) can be determined from a single specimen [2]. However, the presented approach requires three different impedance measurements, resulting from the three electrodes of the specimen. This in turn results in a highly complex, four-terminal impedance measurement setup, as sense and load contacts have to be switched between the three electrodes of the specimen as well as open and short reference measurements. Thus an evolution of the aforementioned approach is developed, with the premise of evaluating only a single frequency-dependent impedance measurement. This is realised by the optimisation of a multi-electrode configuration, in which the electrodes are either connected to the high potential terminals or to the ground terminals of the impedance analyser.

\section{Optimised Electrode Topology}

The configuration of the electrode topology is derived using an iterative approach based on a finite-element model of a piezoelectric disc, which is similar to the sequential element rejection and admission method (SERA) [3]. For the initial electrode configuration, the faces of the disc are fully covered with the high potential and the ground electrodes respectively. Using this initial configuration, frequency-domain simulations yield the frequency- and radius-depended induced electrical charge density $\sigma(f, r)$ for each radial position $r$ on each of the disc's faces. By varying the parameters of the piezoelectric material model, the sensitivity $\Upsilon_{\sigma}\left(f, r, p_{i}\right)$ of the charge density with respect to each material parameter $p_{i}$ is determined. It is assumed that, in order to obtain a high sensitivity of a measured impedance, electrodes should be placed where the sensitivity of the induced charge density is high. To keep axial symmetry, only a one-dimensional, radially dependent criterion value $C(r)$ for the SERA is required for the lower and upper face of the specimen. In the presented procedure, a high potential electrode is placed where $C(r)$ is above a certain threshold value $t$, and a ground electrode is placed where $C(r)$ is below the negative of the same value $(-t)$ : 


$$
\begin{array}{ll}
C(r) \leq-t: & \text { Ground electrode } \\
-t<C(r)<t: & \text { No electrode } \\
C(r) \geq t: & \text { High potential electrode }
\end{array}
$$

As the determined sensitivity of the charge density is dependent on frequency and the respective material parameter as well as on the radius, a method to resolve this additional dependencies is required. Two approaches are presented here. The first one being a simple sum with respect to frequency $f$ and the material parameters $p_{i} \in$ $\left\{c_{11}, c_{12}, c_{13}, c_{33}, c_{44}, \varepsilon_{11}, \varepsilon_{33}, e_{15}, e_{31}, e_{33}\right\}$ :

$$
C(r)=\sum_{f} \sum_{p_{i}} \Upsilon_{\sigma}\left(f, r, p_{i}\right) .
$$

This translates to placing electrodes wherever the overall sensitivity is high. However, one might prefer placing electrodes where the sensitivities of different material parameters each show a different behaviour in frequency domain. One way of determining such a criterion value is based on the determinant criterion [4]:

$$
C(r)=\left|\begin{array}{ccc}
\left\langle\Upsilon_{\sigma}\left(f, r, p_{1}\right), \Upsilon_{\sigma}\left(f, r, p_{1}\right)\right\rangle & \left\langle\Upsilon_{\sigma}\left(f, r, p_{1}\right), \Upsilon_{\sigma}\left(f, r, p_{2}\right)\right\rangle & \ldots \\
\left\langle\Upsilon_{\sigma}\left(f, r, p_{2}\right), \Upsilon_{\sigma}\left(f, r, p_{1}\right)\right\rangle & \left\langle\Upsilon_{\sigma}\left(f, r, p_{2}\right), \Upsilon_{\sigma}\left(f, r, p_{2}\right)\right\rangle & \ldots \\
\ldots & \ldots & \ddots
\end{array}\right| .
$$

The two criteria are smoothed in radial direction using a Gaussian filter to prevent electrodes that are too small to realise. Using the threshold value $t$, the placement of electrodes is determined. The procedure is then repeated using the now modified electrode topology until the topology converges.

\section{Results}

Figure 1 shows the resulting electrode topology when using the sum of sensitivities as a criterion value (a) and the determinant criterion (b), along with the sensitivities of the electrical impedance $\Upsilon_{Z}$ for each of the material parameters. While the first approach yields an overall higher sensitivity with respect to most material parameters, the electrical impedance is nearly independent of $c_{12}$. In contrast, the overall sensitivity of the second approach is slightly lower but varies less over the different material parameters, which is preferable for a material parameter identification process. In addition, the fact that only electrodes with the same potential occur on each side of the specimen facilitates the measurement with standard impedance analysis equipment. In an optimisation procedure similar to the one presented by Jurgelucks et al. [5] the topology of specimen $b$ is parameterised by the four electrode bound radii and optimised based on the sensitivity of the electrical impedance calculated via automatic differentiation. The determinant criterion [4] is again used to define the target function. The resulting electrode configuration (Figure $1 \mathrm{c}$ ) shows a further reduced variance of the sensitivities of the material parameters. The optimised electrode radii for a piezoelectric disc with a radius of $5 \mathrm{~mm}$ and a thickness of $1 \mathrm{~mm}$

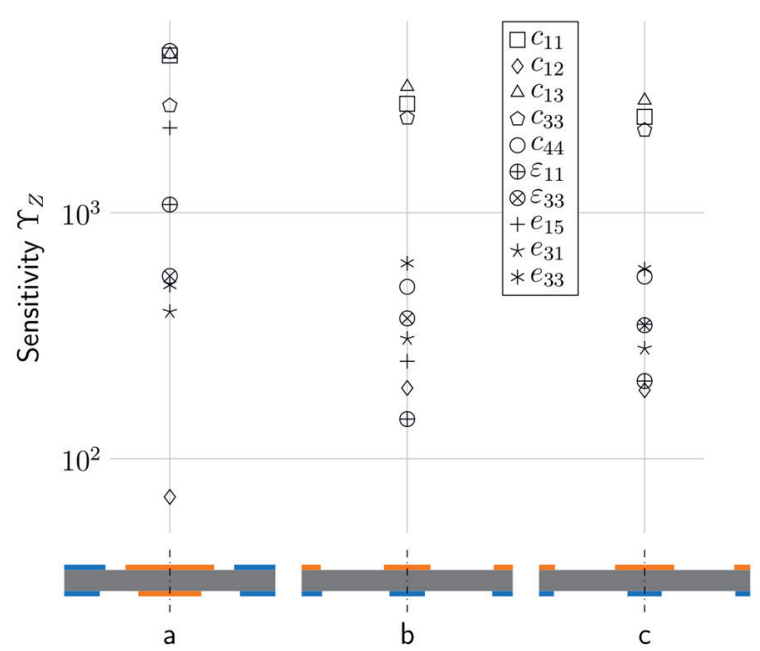

Figure 1: Sensitivities of the electrical impedance for different electrode topologies, generated using the sum of sensitivities (a) and the determinant (b) as criterion value, as well as the optimised configuration of the latter (c). Blue electrodes are on ground, orange electrodes are on high potential.

are $1.4 \mathrm{~mm}$ and $4.3 \mathrm{~mm}$ on the upper face as well as $0.8 \mathrm{~mm}$ and $4.3 \mathrm{~mm}$ on the lower face.

\section{Conclusions}

Applying a combination of the sequential element rejection and admission method and optimisation, an electrode topology for piezoelectric discs is determined. The resulting configuration has two electrodes on both faces of the disc, each with a ring at the outer edge of the disc and a circle at the centre. The impedance of this configuration shows significant sensitivity to all material parameters and is sufficiently simple to be analysed using standard measurement equipment. Using this sample as a specimen the authors aim to realise a full material characterisation procedure based on a single electrical impedance measurement.

\section{References}

[1] American National Standards Institute. IEEE Standard on Piezoelectricity, (1987)

[2] N. Feldmann, V. Schulze, L. Claes, B. Jurgelucks, A. Walther and B. Henning, Inverse piezoelectric material parameter characterization using a single disc-shaped specimen, $t m$ - Technisches Messen (2020); doi: 10.1515/teme2020-0012

[3] O. M. Querin, M. Victoria, C. A. Gordoa, R. Ansola and P. Martí, Topology Design Methods for Structural Optimization (2017); isbn: 978-0-08100916-1

[4] V. V. Fedorov, Theory of Optimal Experiments, (1972); isbn: 0-12-250750-9

[5] B. Jurgelucks, L. Claes, A. Walther and B. Henning, Optimization of triple-ring electrodes on piezoceramic transducers using algorithmic differentiation. Optimization Methods and Software (2018); doi: 10.1080/10556788.2018.1435652 